\title{
A radical rearrangement
}

A new study adds programmed DNA rearrangement to the functional repertoire of RNA. This work - in the unicellular eukaryote Oxytricha trifallax - also adds to our understanding of how RNAs might transmit information across generations.

Ciliates such as O. trifallax have both diploid germline micronuclei and polyploid somatic macronuclei. After fertilization, the macronucleus breaks down and must be reconstituted from the micronuclear genome - this is quite a feat, as the genomes of the two types of nucleus are very different. The information in the micronuclear genome is compressed by $95 \%$, through the deletion of transposons and internally eliminated sequences, and the remaining fragments must be rearranged and reconstituted to form so-called macronuclear nanochromosomes. This process is highly specific and reproducible, suggesting that an accurate mechanism is involved.

One suggestion for how this programmed rearrangement occurs involves a putative RNA cache that is derived from the maternal macronucleus and transmitted to the next generation. This cache could act as a template for the reassembly of the macronuclear genome. Nowacki and colleagues tested this possibility by directing RNAi against the potential components of such a cache that would be required for the correct assembly of two macronuclear genes. In both cases, when the RNAi reagents were fed to parental cells before fertilization, the result was aberrant unscrambling of the genes in the macronuclear genome of the progeny, supporting the cache hypothesis. The authors also demonstrated the existence of RNAs that could fulfil the template role. RT-PCR revealed the presence of long macronuclear transcripts (both sense and antisense) at times that are consistent with a role in rearrangement, but not in vegetative cells.

To test their model directly, Nowacki and colleagues injected sexually reproducing $O$. trifallax cells with synthetic DNA and RNA constructs, with the aim of respecifying the rearrangement pattern in the progeny. Following injection with either artificial chromosomes or synthetic RNA templates, with altered ordering of macronuclear gene segments in both cases, the progeny macronuclei contained the corresponding rearrangements. Notably, these altered conformations can be stably inherited, with transmission to $\mathrm{F}_{2}$ and $\mathrm{F}_{3}$ sexual generations.

The extensive genome rearrangment seen in the formation of the O. trifallax macronuclear genome is clearly the exception rather than the rule. However, this study presents the intriguing possibility that RNA templates may be involved in less widespread rearrangements in other eukaryotes, and suggests a role for RNAs in transgenerational effects. The authors also note that a few mutations (near rearrangement boundaries) in their synthetic templates could be transferred to the rearranged gene, suggesting the intriguing possibility that such RNAs might also direct DNA repair.

Louisa Flintoft

ORIGINAL RESEARCH PAPER Nowacki, M. et al.

RNA-mediated epigenetic programming of a genome-rearrangement pathway. Nature 28 November 2007 (doi:10.1038/nature06452) FURTHER READING Matzke, M. A. \& Birchler, J. A. RNAi-mediated pathways in the nucleus. Nature Rev. Genet. 6, 24-35 (2005)

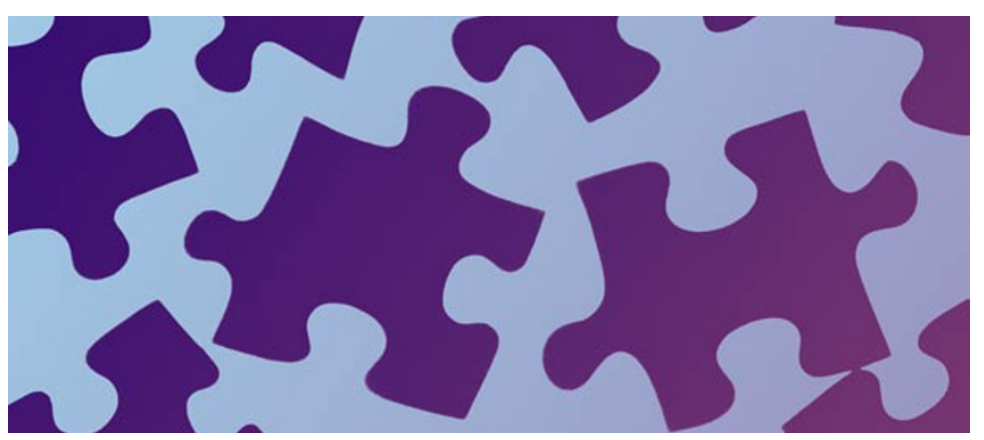

